УДК 304.2

$10.17213 / 2075-2067-2021-1-259-268$

\title{
О ВЗАИМОДЕЙСТВИИ МЕЖДУ СОЦИАЛЬНЫМИ НАУКАМИ И МАТЕМАТИКОЙ
}

\author{
(C) 2021 г. А. В. Дятлов, К. В. Воденко
}

\section{Южно-Российский государственный политехнический университет (НПИ) имени М. И. Платова, г. Новочеркасск, Россия}

Статья посвящена изучению специфики взаимодействия между социальными науками и математикой. Авторы исходят из необходимости объяснения вопросов подготовки к технологическому перевооружению и осуществлению перехода к экономике знаний в контексте изучаемой проблематики. Анализируются вопросы, связанные с вероятностными моделями в сочиальных науках, ичиклиность в глобальной сочииальной динамике, вопросы технологического перевооружения в условиях перехода к инновационной экономике.

Ключевые слова: социальные науки; методология; математика; модели; инновации.

\section{ON THE INTERACTION BETWEEN SOCIAL SCIENCES AND MATHEMATICS}

\section{(C) 2021 A. V. Dyatlov, K. V. Vodenko}

\section{Platov South Russian State Polytechnic University (NPI), Novocherkassk, Russia}

The article is devoted to the study of the specifics of the interaction between social sciences and mathematics. The authors proceed from the need to explain the issues of preparation for technological re-equipment and the implementation of the transition to the knowledge economy in the context of the studied problem. The issues related to probabilistic models in social sciences, cyclicity in global social dynamics, and issues of technological re-equipment in the transition to an innovative economy are analyzed.

Key words: social sciences; methodology; mathematics; models; innovations. \section{статью \\ Что послужило причиной написать эту}

В то время как в развитых западных государствах, а также в быстрорастущих восточных странах математика широко используется в социальных науках, то в России все еще существуют предубеждения. Многие математики считают, что не стоит заниматься моделированием социальных процессов при наличии многих других областей исследований в чистой и прикладной математике [8]. С другой стороны, многие ученые-исследователи социальных наук придерживаются мнения, что математика не может описать социальные процессы во всей их сложности и что только чисто качественный анализ приводит к лучшим результатам. Из-за этих доминирующих до настоящего времени мнений наблюдается опасное отставание в развитии математического моделирования социальных структур, систем и процессов в России. По нашему мнению, пришло время исправить такое мнение об использовании математики для моделирования в социальных науках. Чтобы помочь в этой коррекции, обсудим некоторые классические и новые приложения матема- 
тики для моделирования социальных структур, систем и процессов. Кроме того, мы подчеркнем известную как западную, так и уже восточную истину о том, что надвигающаяся восходящая часть следующего цикла Кондратьева - это наиболее подходящее время для перехода от экономики, основанной на экспорте сырья ,через цифровизацию к экономике, основанной на знаниях. Для подготовки этого перехода у нас мало времени, да и оно пройдет очень быстро.

\section{Некоторые аспекты роли математики в социальных науках}

Применение математики в социальных науках тесно связано с измерениями. Мы считаем, что математические исследования и качественный анализ должны идти рука об руку с социальными науками, что прекрасно иллюстрируется процессом измерений - при измерении количеств нам нужен качественный анализ. Прежде чем мы начнем измерять что-либо, связанное с каким-либо объектом или субъектом, мы должны провести качественный анализ, чтобы определить величины, измерение которых будет способствовать решению задачи, в связи с которой мы делаем измерения. Кроме того, следует также выбрать методологию измерения. Затем после проведенных измерений снова необходимо провести качественный анализ адекватности полученных результатов в отношении решаемой задачи [4]. Мы охватим тему измерений, потому что через нее мы быстро войдем в поле, связанное с вопросом о различиях в измерении и статистическом описании, используемых в естественных и социальных науках.

\section{1. О различиях в измерениях в естест-} венных и социальных науках

Что касается методики измерений, то у нас есть 4 типа измерительных шкал:

1) номинальная шкала - разграничение между исследуемыми объектами производится только на основе свойств классов, к которым принадлежат эти объекты. Примерами таких шкал являются различия по языку, национальности, этнической принадлежности и т.д.;

2) ранговая шкала - здесь в дополнение к различию между объектами происходит и определение порядка между ними по отношению к измеряемому свойству;
3) интервальная шкала - дополнительно к различению и расположению вводится и степень разницы между объектами на основе сопоставления присущих объектам значений измеряемой характеристики (свойства). Примером такой интервальной шкалы является температура, измеренная в градусах Цельсия;

4) шкала отношений - в дополнение ко всему, что связано с другими породами, при измерении по этой шкале также могут образовываться отношения между значениями соответствующей величины, характеризующей свойство для каждых двух измерений объекта. Примером такой шкалы является шкала температуры, измеряющая температуру в градусах Кельвина. При наличии шкалы, измеряющей температуру в градусах Цельсия, если один объект имеет температуру 20 градусов, а другой объект - температуру 40 градусов, мы не можем сказать, что второй объект в два раза теплее первого. Чтобы сформировать отношение, мы должны перейти к шкале абсолютной температуры, измеряющей температуру в градусах Кельвина. Тогда первый объект имеет температуру 293 градуса Кельвина, а второй - 313 Кельвинов, и этот второй объект в 313/293 = 1,0682 раз теплее, чем первый.

На основе четырех шкал у нас есть два типа измерений:

- качественные измерения - используется номинальная и (или) ранговая шкалы;

- количественные измерения - используется интервальная и (или) шкала отношений.

В естественных науках в основном проводятся количественные измерения. В социальных науках также очень распространены качественные измерения.

\section{2. О различиях в вероятностном описа-} нии в естественных и социальных науках

Многие системы в природе и обществе являются недетерминированными по своей сущности, или у нас недостаточно информации, чтобы точно предсказать, как будут развиваться такие системы, даже если они детерминированы, поэтому для таких процессов и систем используется вероятностное описание. И здесь возникает существенный вопрос: различается ли вид вероятностного описания 
систем в естественных науках от вида вероятностного описания систем в социальных науках? Ответ - да, доминантный вид вероятностного описания для одного и другого типа систем различен. И это имеет существенные последствия. Доминантное описание в естественных науках, основанных на распределении Гаусса (которые так часто встречаются, что их называют нормальными). Доминантный тип вероятностного описания в социальных науках основан на негауссовском распределении. Что из этого следует?

1. Большая часть гауссовского распределения величины в естественнонаучных системах сосредоточена в узкой области вокруг среднего значения распределения. Это позволяет таким естественнонаучным системам переходить от вероятностного к детерминированному описанию и, следовательно, разрабатывать подробные, но относительно простые модели поведения таких систем. При негауссовом распределении нет такой концентрации значений величины вокруг среднего значения. Следовательно, переход к детерминированному описанию невозможен.

2. На социальные системы действует гораздо больше факторов по сравнению с количеством факторов, влияющих на естественнонаучные системы. Не вдаваясь в подробности, можно сказать, что распределение, соответственно, факторов в естественнонаучных системах является гауссовым, а в большинстве социальных систем - негауссовым. И опять же, существенно больше значений в гауссовых распределениях находится в узком интервале, что означает относительно небольшое количество факторов, существенно влияющих на систему. В социальных системах большинство значений распределения занимают существенно более широкий интервал, это означает, что гораздо больший набор факторов влияет на соответствующие системы. Таким образом, в то время как в естественнонаучных системах обычно следует учитывать небольшое количество факторов, в социальных системах должно учитываться гораздо большее количество влияющих на них факторов.

\section{Вероятностные модели в социальных} науках

Человеческое общество - открытая система, элементами которой являются люди.
Система открыта, потому что существует не только взаимодействие между ее элементами, но и взаимодействие с окружающей природной и технологической средой. Элементы системы - люди, которые могут находиться в разных физических и психических состояниях, эти состояния могут меняться. Эти перемены лежат в основе динамики социальных систем, которую можно исследовать на основе соответствующих глобальных переменных. Для этих переменных могут быть написаны модельные уравнения, чтобы дать нам информацию о будущем поведении системы.

Сразу следует отметить, что элементы социальных систем - люди, которые сами являются довольно сложными системами, имеют мысли, эмоции, намерения и взаимодействуют несколькими способами. И еще: организации и самоорганизации в обществе часто происходят на основе человеческого интеллекта, в связи с чем они лучше или хуже планируются, в то время как в естественнонаучных системах организации и самоорганизации происходят как следствие природных законов, действующих на элементы, которые не имеют интеллекта.

Математическое описание социальных процессов строится на следующем представлении о людях и обществе. Люди функционируют так, что различные рациональные рассуждения и эмоции, в конце концов, формируются, как мнения, например, за какую партию голосовать или в какой стране провести летние каникулы. Другие рассуждения, эмоции и идеи могут привести к изменению мнения человека, то есть к переходу от одного мнения к другому. Кроме того, могут возникнуть совершенно новые взгляды, и поэтому у нас есть постоянно обогащающееся пространство мнений. От микропространства мнений можно перейти к концепции социальной конфигурации, которая описывает распространение мнений в общественных группах, параметрами этой социальной конфигурации являются макроскопические параметры, характеризующие общество. Задача математической социальной динамики заключается в исследовании закономерностей развития социальной конфигураций [8].

Математическая социальная динамика основана на нелинейных уравнениях, и эта не- 
линейность отражает тот факт, что индивиды со своими действиями способствуют созданию и поддержанию коллективного «цивилизационного поля», которое имеет экономические, политические, религиозные, социальные и культурные части. Это поле является важным параметром для стабильности социальной системы и важной характеристикой фазы, в которой находится эта система. Взаимодействие между коллективным полем и индивидами довольно сильное, поле может ориентировать деятельность индивидов, активизировать их потенциальные мнения и действия. В конце концов, поле и индивиды находят какое-то квазиравновесное состояние, в котором общество находится некоторое по продолжительности время. В этом состоянии общество имеет определенную самосогласованную коллективную структуру, а его развитие может быть в некоторой степени предсказано с вытекающими последствиями. Предсказуемость должна быть уменьшена там, где параметры, контролирующие эволюцию социальной структуры, достигают критических значений (в результате взаимодействий, которые являются внутренними или внешними для структуры), таких критических значений, при которых появляются альтернативные пути развития. При таком состоянии небольшие колебания (например, действия группы влиятельных людей) могут привести к изменению пути социального развития. Это изменение может быть революционным.

Чтобы не быть голословными, рассмотрим одну иллюстрирующую модель. Пусть есть некое общество, и рассматриваем эволюцию общественного мнения по какому-то вопросу, по которому есть две возможных альтернативы: скажем, останется Крым в составе Украины или присоединится к России. Пусть в обществе есть $2 N$ индивидов, и $n_{1}$ из них поддерживают первое мнение, а $n_{2}$ - второе мнение. Так $n_{1}+n_{2}=2 N$, и если $n_{1}=N+n$, то $n_{2}=N-n$. Другими словами, чтобы описать эволюцию общественного мнения по этому вопросу, мы должны исследовать динамику переменной $n(t)$. Если предположить, что за интересующий нас временной интервал население исследуемой территориальной единицы не изменяется, то исследование эволюции $n(t)$ эквивалентно изучению эволюции переменной $x(t)=n(t) / N$.
Значения переменной $x(t)$ со временем изменяются, потому что часть людей меняет свое мнение. Различные типы факторов могут воздействовать на эту переменную, и эти факторы можно разделить на две группы. К одной группе отнесем существенные и постоянно действующие факторы, устанавливающие тенденцию к изменению мнения. К другой группе - относительно слабые факторы, действующие на относительно небольшом временном интервале. Действие этих факторов обозначим как колебания. Таким образом, эволюция переменной $x(t)$ описывается уравнением:

$$
\frac{d x}{d t}=\alpha(x)+\beta(x) \xi(t),
$$

где $\alpha(x)$ учитывает влияние факторов, которые задают тренд, а $\beta(x)$ учитывает влияние факторов, ответственных за интенсивность флуктуаций. Переменная $\xi(t)$ называется случайной силой и учитывает суммарное действие случайных флуктуаций. Уравнение (1) называется уравнением Ланжвена [3].

Во многих случаях $\xi(t)$ может аппроксимироваться с гауссовой $\delta$-коррелирующей случайной силой со средним значением 0 и автокорреляционной функцией $[\xi(t+\tau) \xi(t)]$ $=\delta(\tau)$, где $[\ldots]$ означает среднее всевозможных случайных сил. Для случайной силы этого вида, также называемой гауссовым белым шумом, уравнение Ланжвена может быть сведено к уравнению Фокера-Планка:

$$
\begin{gathered}
\frac{\partial P(x, t)}{\partial t}=-\frac{\partial}{\partial x}[K(X) P(x, t)]+ \\
+\frac{\varepsilon}{2} \frac{\partial^{2}}{\partial x^{2}}[Q(x) P(x, t)],
\end{gathered}
$$

где

$$
\begin{gathered}
\alpha(x)=K(x)-\frac{\varepsilon}{4} \frac{\partial Q}{\partial x} \\
\beta(x)=\varepsilon^{1 / 2} Q(x)^{1 / 2} \\
P(x, t)=N p(n, t)
\end{gathered}
$$

где $p(n, t)$ — это вероятность общества иметь социальную конфигурацию, описываемую $n$ в момент времени $t$.

Исследования уравнения (3) показывают, что со временем $P(x, t)$ приближается к независимому от времени распределению, заданному 


$$
\begin{gathered}
P_{s}(x)= \\
=P_{s}(0) \frac{Q\left(x_{0}\right)}{Q(x)} \exp \left[\frac{2}{\varepsilon} \int_{x_{0}}^{x} d x^{\prime} \frac{K\left(x^{\prime}\right)}{Q\left(x^{\prime}\right)}\right],
\end{gathered}
$$

где

$$
\begin{gathered}
P_{s}(0)=\frac{1}{A Q\left(x_{0}\right)} ; \\
A=\int_{-1}^{1} d x^{\prime}\left(\frac{B}{Q\left(x^{\prime}\right)}\right) ; \\
B=\exp \left[\frac{2}{\varepsilon} \int_{x_{0}}^{x^{\prime}} d x^{\prime \prime} \frac{K\left(x^{\prime \prime}\right)}{Q\left(x^{\prime \prime}\right)}\right],
\end{gathered}
$$

Из (4) и $P(x)=N p(n)$ мы находим то, что нас интересует. Ожидаемое значение $n$ вычисляется как

$$
\bar{n}=\int(n),
$$

и заинтересованные политики смогут сделать расчеты, какие действия предпринимать.

\section{Социальные сети и теория графов}

Другое применение математического аппарата связано с теорией сетей. Социальные сети являются важным элементом социальной структуры. Эти сети могут быть представлены различными способами, например, через матрицы или через графы. Наиболее распространенным представлением социальных сетей являются графы, узлы которых соответствуют индивидуумам и организациям, а связи между узлами - связям между людьми и органами.

Многое можно узнать об индивидуумах, изучая социальные сети, в которых участвуют эти люди. Соответственно, новое исследовательское направление называется анализом социальных сетей. Анализ социальных сетей широко используется, например, в эпидемиологии, чтобы лучше понять распространение заражения (пандемии). Интернет является средой для размещения многих социальных сетей.

Сети и, в частности, социальные сети имеют разные математические характеристики. Например, мы можем говорить о траектории в сети (совокупность узлов и связи между ними). Мы можем исследовать пути в его эквивалентность первому определению (траектория между двумя узлами, которая не проходит дважды через один и тот же узел). Существуют также циклы - это пути, для которых исходный и конечный узел совпадают. Сети имеют диаметр (самое большое расстояние между двумя узлами). С узлами сети также может быть связано понятие «мощность» (также называемое властью). Узлы имеют несколько характеристик, некоторые из них:

- степень - количество соединений, которые выходят из данного узла;

- близость - среднее количество «переходов» из данного узла ко всем другим узлам в сети;

— промежуточность - количество отрезков между промежуточными узлами, в которых нуждается узел, чтобы достичь любого по других узлов самым коротким путем;

- мощность (власть) узла зависит от количества самых коротких путей в сети, которые проходят через этот узел.

Мощность вычисляется по формуле:

$$
B_{k}=\sum_{(i, j) \in E} \frac{P_{k}(i, j)}{(n-1)(n-2) P(i, j)},
$$

где $P(i, j)$ - количество кратчайших путей между узлами $i$ и $j ; P_{k}(i, j)$ - количество кратчайших путей между узлами $i$ и $j$, проходящими через узел $k ; n-$ количество узлов в сети.

Примером хорошей ситуации в социальной сети является положение Медичи среди других богатых семей во Флоренции. Медичи не являются самой богатой семьей, но их мощность в флорентийской социальной сети богатых семей является самой большой и в 2 раза больше мощности следующей семьи. И с хорошим положением в социальной сети пришли большие богатства и большая власть.

Аналогичным образом рос и восходил Борджиа, как мелкий благородный род из периферии испанской благородной сети в целом. Единственным способом добраться до центральной позиции в сети был путь возведения не в благородной, а в церковной иерархии. Стратегия, которой неустанно следовали Борджиа, позволила им добраться до центрального места в средневековых социальных сетях, освятив двух пап из своих рядов.

\section{Цикличность в глобальной социаль- ной динамике}

Давайте теперь обсудим применение методов математики в моделировании глобаль- 
ной экономической и социальной динамики. Почему мы останавливаемся именно на этом приложении, станет ясно далее по тексту. Модели глобальной динамики начали развиваться профессором Массачусетского технологического института Джеем Райтом Форрестером по заказу римского клуба в начале семидесятых годов XX века [5]. Д. Форрестер отслеживал следующие основные процессы в своей модели:

- быстрый рост населения;

- индустриализация и связанный с ней рост промышленного производства;

- увеличение промышленных отходов, приводящих к загрязнению окружающей среды;

- недостаточное количество пищи для пропитания населения мира;

- ограничение природных ресурсов сырья.

Модель Форрестера предсказывает кризис человечества в XXI веке из-за ограниченности природных ресурсов. Поскольку природные ресурсы могут только уменьшаться, кризис будет углубляться, если технологическая база человечества не включит новые технологии, более эффективно расходуя ограниченные природные ресурсы [6].

Следующий шаг в моделировании мировой динамики связан с моделью М. Месаровича и Э. Пестеля. В этой модели все страны мира делятся на 10 регионов, каждый регион описывается моделью, имеющей одну и ту же блочную структуру для всех регионов. Связь между регионами осуществляется путем миграции населения и товарообмена. Региональные модели содержат экономическую, демографическую и энергетическую часть. Модель Месаровича-Пестеля напрямую не предсказывает глобальную катастрофу. Вместо этого модель предсказывает серию региональных катастроф, которые могут превратиться в глобальную катастрофу, если не будут приняты меры.

А теперь давайте сосредоточим наше внимание на цикличности в глобальной динамике, потому что эта цикличность тесно связана с наукой и инновациями. Наука и инновации являются основными движущими силами общественного развития. И процессы в науке и инновациях тоже имеют циклический характер. Как следствие, происходят циклические колебания экономической активности. Различные типы экономических циклов имеют разный период. Возможно, самый известный из этих циклов с периодом около полувека был обоснован русским экономистом Николаем Кондратьевым и называется циклом Кондратьева [1]. Основываясь на наблюдениях Кондратьева, Шумпетер предположил, что циклический характер инновационных процессов может вывести экономическую систему из одного равновесного состояния и подвести ее к новому равновесному состоянию, которое просуществует до тех пор, пока не иссякнут соответствующие инновации, после чего возникнет экономический и социальный кризис. Этот кризис вызовет следующую инновационную волну, которая, в свою очередь, приведет к переходу экономической системы в новое состояние. Кризис направляет экономических агентов к инновациям, которые они должны принять, чтобы выжить. Усиленная тяга к инновациям влечет за собой появление кластера инноваций, приводящих к появлению новых экономических отраслей, которые ведут к окончанию и началу нового цикла Кондратьева. Таким образом, технологические кластеры с их появлением, развитием, распространением и истощением потенциала воздействия на экономику приводят к росту валового внутреннего продукта развитых стран по линии, описываемой циклическими колебаниями вокруг (на данный момент с 2018 года) восходящей тенденции.

Итак, экономический рост связан с техническим прогрессом, а технический прогресс зависит от интеллектуального капитала. Интеллектуальный капитал существенно зависит от численности квалифицированного трудоспособного населения. Сегодня мы живем в интересные времена демографического перехода (от роста населения к стабилизации числа людей), этот демографический переход в развитых странах закончится примерно в 2040 году и в развивающихся странах продлится примерно до 2100 года. Здесь мы отмечаем, что население Земли является важным параметром, под изменения которого подстраиваются социальные, экономические, культурные и исторические процессы на Земле [7]. Итак, в конце демографического перехода население Земли составит около 10,5 миллиардов человек, 1,5 миллиарда из которых будут в совре- 
менных развитых странах. Затем, примерно в 2200 году (если не произойдет самоуничтожение или экстремальные события, такие, как, например, падение большого метеорита), население Земли упадет до 5,2 миллиарда человек, 1,2 миллиарда из которых будут жить в Китае и 1 миллиард - в Индии.

Но давайте вернемся к экономике и предположим, что у нас есть технологически отсталое государство без большого населения. Существует два способа, которыми технологически отсталая страна может наверстать отставание: разработка собственных технологий и использование иностранных технологий. В начальный период догоняющая национальная научно-инновационная система слаба и поэтому опирается на импорт технологий. Постепенно производство собственных технологий берет верх, и на втором догоняющем этапе соответствующая сторона уже гораздо больше полагается на свою собственную научно-технологическую систему. Грубая ошибка заключается в том, чтобы полагаться только на зарубежные технологии и не развивать национальную научно-техническую систему. В таком сценарии нет второго догоняющего этапа, и с этого момента соответствующее государство начинает снова отставать, потому что оно зависит от зарубежных стран, не предоставляющих технологий, с помощью которых государство отнимет у них первенство и захватит значительную часть рынков. Типичным примером такой грубой ошибки является Россия. Почти два десятка лет в России преобладал взгляд Егора Гайдара, что российская наука бесполезна и, если России нужна наукоемкая продукция, наша страна будет покупать ее на Западе. В результате таких «идеологов» российская наука систематически подавлялась, русские ученые были буквально изгнаны на Запад, а Россия была доведена практически до положения сырьевого придатка западных экономик. Но нашелся кто-то, кто вытесняет эти «идеи» из российского интеллектуального пространства, и результаты изменений начали быстро ощущаться.

Давайте скажем несколько слов о расчетах моделей мировой динамики до 2050 года. Модели говорят нам, что в отсутствие существенных глобальных потрясений крупнейшей экономикой в 2050 году будет экономика
Китая, а за второе место будут сражаться Индия и США [2]. Иностранные модели дают приоритет Индии, но наши расчеты поддерживают США, которые с 2018 года снова вошли в период экономического подъема. Кстати, примерно с 2018 года начинается следующий цикл Кондратьева, в течение которого полностью будет развиваться потенциал технологий, возникших в текущем цикле электроника на основе нанотехнологий, биотехнологии, информационные и коммуникационные технологии, и, как говорят немцы, место на солнечной стороне жизни будет для тех народов, которые успешно используют возможности этих технологий [6].

За технологическое перевооружение и переход к инновационной экономике

Какой стратегии мы должны следовать, чтобы быстро начать сокращать отставание от развитых держав? Наши рассуждения и расчеты говорят об этом [4]. Одновременно нам предстоит решить три группы проблем развития:

- демографические проблемы и проблемы, связанные с человеческим капиталом;

- проблемы модернизации экономики;

- проблемы, связанные с переходом к инновационной экономике (экономика, основанная на знаниях).

В частности:

1) требуется реиндустриализация, вместе с этим следует быстро повысить технологический уровень промышленности;

2) требуется технологическое перевооружение непромышленных отраслей экономики на основе высоких и средних технологий. Например, в сельском хозяйстве сегодня высокими технологиями являются биотехнологии, связанные с растениями, животными и лекарствами, а также генная инженерия, среднесрочные технологии, связанные с микроорганизмами или удобрениями;

3) необходимо осуществлять переход от состояния низкотехнологичного уровня экономики и низкой инновационной активности к состоянию среднетехнологического уровня экономики и средней инновационной активности;

4) параллельно с использованием иностранных технологий необходимо укрепить национальную научно-инновационную систе- 
му, чтобы начать производство стабильного потока национальных технологий;

5) социальное неравенство негативно влияет на экономическое развитие, поэтому для ускорения экономического развития необходимо работать над сокращением социального неравенства, но при этом необходимо помнить, что сказали классики экономики: «Каждому — по труду!»;

6) необходимо остановить демографическую катастрофу, сокращение человеческого капитала и его отток за границу. Отметим, что именно по этим вопросам написано довольно много статей. По нашему мнению, судить, является Россия умелым политиком или нет, можно по тому, как она решает вопросы, связанные с демографией и человеческим капиталом;

7) особые личные усилия должны быть предприняты для снижения дефицита квалифицированных ученых и квалифицированных инженерно-технических кадров;

8) быстрый экономический рост достигается за счет производства высокотехнологичной инновационной продукции с высокой добавленной стоимостью. По этому пути развития шли такие страны, как Сингапур, Израиль или Финляндия.

\section{Выбор российской элиты сегодня: РАН или ... \\ Итак, начался запуск следующего, шесто-} го, цикла Кондратьева [1]. Движение в положительном направлении начинает уже чувствоваться. Азбучная истина в современной теории технологического развития состоит в том, что восходящая часть цикла Кондратьева - это время, когда отставшие страны с помощью соответствующей научно-технологической политики, возможно, быстро вышли бы из плена технологической и социальной отсталости. Восходящая часть начавшегося цикла Кондратьева будет между 2018 и 2035 годами. У нас есть время, чтобы подготовить почву для относительно долгого процесса массивного технологического перевооружения и перехода к экономике, основанной на знаниях. Но на данный момент сделано очень мало, а инновации - это то, что преодолевает кризисы, депрессии и отсталости. Какие области критичны для быстрого экономического развития технологий? Области нанотехнологий, биотехнологий и генной инженерии, компьютерных или, более обще говоря, информационно-коммуникационных технологий, водородной энергетики и других форм альтернативной энергетики. А может ли Россия идти в ногу? Да, может! У нашей страны есть инструмент для решения этой важной задачи - это система институтов РАН, но они должны быть существенно усилены учеными, для которых необходимо создать отличные условия для работы и общественного статуса (соответственно и достойная оплата труда). Если этого не сделать, цикл Кондратьева начнется и пройдет, а отставание России будет продолжаться. Другими словами, еще более уверенными шагами мы пойдем к банановой республике, основанной на вывозе леса, рыбы и своих природных ресурсов.

Итак, две альтернативы развития России: РАН (инновации и экономика, основанная на знаниях) или сырьевой придаток. Выбор должен быть сделан сейчас, потому что следующий подходящий момент для ускоренного догоняющего развития будет примерно через 50 лет. И если сегодня российская элита выбирает нефть и газ (что не так уж маловероятно), то через 50 лет из развитых стран с усмешкой будут наблюдать, как одна сырьевая колония будет грызть себя, чтобы сделать технологический скачок. Для того чтобы не дойти до такой жалкой ситуации, мы посылаем четкое предупреждение. И оно вовремя.

\section{Литература}

1. Kondratieff N.D. Die langen Wellen der Konjuktur // Archiv fuer Sozialwissenschaft und Sozialpolitik. - 1926. - №56 (3). Pp. 573-609.

2. Knoke D. Political networks. The structural perspective. Cambridge University Press. Cambridge, 1990.

3. Mensch G. Stalemate in technology Innovations overcome depression. - Ballinger, New York, 1979.

4. Hirooka M. Innovation dynamism and economic growth. A nonlinear perspective. Cheltenham, UK, 2006.

5. Pecci A., Pestel E., Mesarovich M. Der Weg INS 21. Jahrhundert. Club of Rome. Al- 
ternative Strategien fuer die Industriegesellschaft. — Molden Verlag, Wien, 1987.

6. Schumpeter J.A. Business cycles. - McGraw-Hill, New York, 1939.

7. Turchin R. Historical dynamics: Why states rise and fall. - Princeton University Press, Princeton, New Jersey, 2003.

8. Weidlich $W$. Sociodynamics — a systematic approach to mathematical modeling in social sciences. - Dover, New York, 2006.

\section{References}

1. Kondratieff N.D. Die langen Wellen der Konjuktur // Archiv fuer Sozialwissenschaft und Sozialpolitik. - 1926. — №56 (3). Pp. 573-609.

2. Knoke D. Political networks. The structural perspective. Cambridge University Press. Cambridge, 1990.
3. Mensch G. Stalemate in technology Innovations overcome depression. - Ballinger, New York, 1979.

4. Hirooka $M$. Innovation dynamism and economic growth. A nonlinear perspective. Cheltenham, UK, 2006.

5. Pecci A., Pestel E., Mesarovich M. Der Weg INS 21. Jahrhundert. Club of Rome. Alternative Strategien fuer die Industriegesellschaft. - Molden Verlag, Wien, 1987.

6. Schumpeter J.A. Business cycles. - McGraw-Hill, New York, 1939.

7. Turchin R. Historical dynamics: Why states rise and fall. - Princeton University Press, Princeton, New Jersey, 2003.

8. Weidlich $W$. Sociodynamics — a systematic approach to mathematical modeling in social sciences. - Dover, New York, 2006.

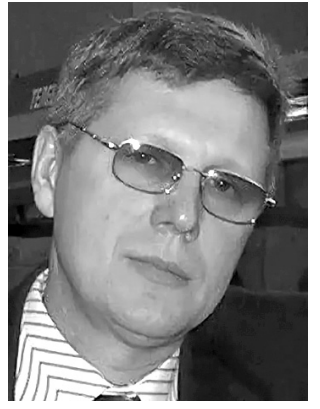

Дятлов Александр Викторович - доктор социологических наук, профессор Института социологии и регионоведения Южного федерального университета.

Dyatlov Alexander Viktorovich - Doctor of Sociological Sciences, Professor, Institute of Sociology and Regional Studies, Southern Federal University.

346500 , г. Ростов-на-Дону, ул. Пушкинская, 160 160 Pushkinskaya st., 346500, Rostov-on-Don, Russia E-mail: avdyatlov@yandex.ru 


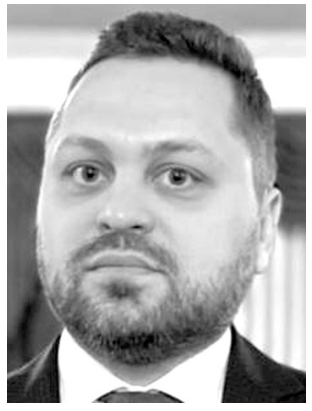

Воденко Константин Викторович - доктор философских наук, профессор, советник при ректорате Южно-Российского государственного политехнического университета (НПИ) имени М.И. Платова. Руководитель научно-образовательной школы «Управление социальными процессами в поликультурном регионе». Сфера научных интересов - социально-культурные и социально-экономические институты и процессы в условиях формирования инновационной модели общественного развития. Руководитель многочисленных прикладных социологических исследований.

Vodenko Konstantin Victorovich - Doctor of Philosophical Sciences, Professor, Adviser to the rector's office, Platov South Russian State Polytechnic University (NPI). Head of the scientific and educational school «Management of social processes in a multicultural region». Research interests - socio-cultural and socio-economic institutions and processes in the formation of an innovative model of social development. Head of numerous applied sociological studies.

346428 , г. Новочеркасск, ул. Просвещения, 132

132 Prosveshcheniya st., 346528, Novocherkassk, Russia

E-mail: vodenkok@mail.ru 